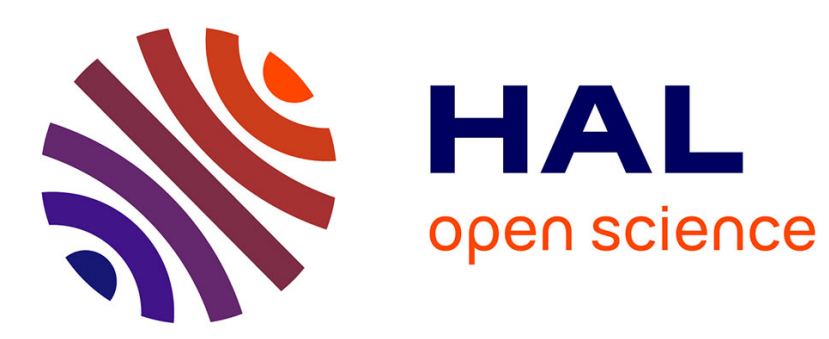

\title{
A Note on the Column Elimination Tree
}

\author{
John R. Gilbert, Laura Grigori
}

\section{To cite this version:}

John R. Gilbert, Laura Grigori. A Note on the Column Elimination Tree. [Technical Report] RT-0251, INRIA. 2001, pp.7. inria-00069923

\section{HAL Id: inria-00069923 https://hal.inria.fr/inria-00069923}

Submitted on 19 May 2006

HAL is a multi-disciplinary open access archive for the deposit and dissemination of scientific research documents, whether they are published or not. The documents may come from teaching and research institutions in France or abroad, or from public or private research centers.
L'archive ouverte pluridisciplinaire HAL, est destinée au dépôt et à la diffusion de documents scientifiques de niveau recherche, publiés ou non, émanant des établissements d'enseignement et de recherche français ou étrangers, des laboratoires publics ou privés. 


\section{A Note on the Column Elimination Tree}

John R. Gilbert , Laura Grigori

\section{$\mathbf{N}^{\circ} \mathbf{0 2 5 1}$}

Août 2001

THÈME 1 



\title{
A Note on the Column Elimination Tree
}

\author{
John R. Gilbert *, Laura Grigori ${ }^{\dagger}$ \\ Thème 1 - Réseaux et systèmes \\ Projets RESEDAS \\ Rapport technique $\mathrm{n}^{\circ} 0251$ - Août $2001-7$ pages
}

\begin{abstract}
This short communication considers the LU factorization with partial pivoting and shows that an all-at-once result is possible for the structure prediction of the column dependencies in $L$ and $U$. Specifically, we prove that for every square strong Hall matrix $A$ there exists a permutation $P$ such that every edge of its column elimination tree corresponds to a symbolic nonzero in the upper triangular factor $U$. In the symbolic sense, this resolves a conjecture of Gilbert and $\mathrm{Ng}[6]$.
\end{abstract}

Key-words: sparse unsymmetric matrices, Gaussian elimination, partial pivoting, structure prediction, column elimination tree

* The work of this author was performed during a visit to CERFACS, Toulouse, France. Copyright (c) 2001 by Xerox Corporation.

† LORIA - Université Henri Poincaré, Vandoeuvre-lès-Nancy, France. 


\section{Note sur l'arbre d'élimination par colonnes}

Résumé : Cette note traite de la factorisation LU avec pivotage partiel et montre qu'un résultat toutesensemble est possible pour la prédiction de dépendances entre les colonnes de $L$ et $U$. Nous montrons que pour chaque matrice carrée $A$ satisfaisant la propriété Hall-forte, il existe une permutation $P$ telle que chaque arête de l'arbre d'élimination par colonnes correspond à un élément non nul symbolique dans le facteur triangulaire supérieur $U$. Dans un sens symbolique, ceci résoud une conjecture de Gilbert et $\mathrm{Ng}$ [6].

Mots-clés : matrices non-symétriques creuses, élimination de Gauss, pivotage numérique, prédiction de structure, arbre d'élimination par colonnes 


\section{Introduction}

Sparsity in matrix computations offers opportunities to save memory space by storing only non-zero elements, shorten execution times by eliminating computations on zeros and exploit parallelism exposed by independent non-zero structures. Exploiting these opportunities often relies on a symbolic computation phase that predicts as accurately as possible which elements will have or can have nonzero values during the numerical computation itself, based only on the nonzero structure of the input matrix.

In LU factorization with partial pivoting, a square matrix $A$ is factored as $P A=L U$, where $P$ is a permutation matrix that depends on the values of the nonzeros of $A$ and cannot be predicted only from the nonzero structure of $A$. Two structure prediction questions have been studied for this problem. The first is to predict bounds on the nonzero structure of the factors $L$ and $U$. The second is to predict which columns of $L$ and $U$ depend directly or indirectly on which earlier columns. We restrict our attention to the class of matrices that satisfy an irreducibility condition called the strong Hall property.

George and $\mathrm{Ng}$ [4] developed upper bounds on the nonzero structure of $L$ and $U$ by employing a row merge graph. Gilbert and Ng [6] showed that this upper bound is as tight as possible in what they called "the exact sense." This means that, given the nonzero structure of a strong Hall matrix, for every edge in the row merge graph there is a choice of values for the nonzeros of $A$ and a pivoting permutation $P$ such that the corresponding element of $L$ or $U$ is nonzero. This is a one-at-a-time result [6]: any single position in the predicted structure can be made nonzero, but it may be the case that no single choice of nonzero values makes all the predicted elements nonzero at once.

The column elimination tree is a tree whose vertices are the columns of $A$, and whose edges correspond to potential dependencies between columns. (A complete definition is below.) Gilbert and $\mathrm{Ng}[6]$ showed that if $k$ is the parent of $j$ in the column elimination tree of a strong Hall matrix $A$, there exists a choice of nonzero values of $A$ that will make column $j$ update column $k$ during factorization with partial pivoting - that is, a choice of nonzero values for $A$ that will make $u_{j k} \neq 0$. This is again a one-at-a-time result.

A stronger statement would be an all-at-once result, showing that all the predicted positions can be made nonzero for the same input values. Unlike the case of sparse QR factorization, no tight all-at-once prediction is possible for the structure of $L$ and $U$. The purpose of this short communication is to show that if we consider only the edges of the column elimination tree, an all-at-once result is possible in the symbolic sense. We prove that for every square strong Hall matrix $A$, there exists a permutation $P$ such that every edge of the column elimination tree corresponds to a symbolic nonzero in the upper triangular factor $U$ of $A$ with partial pivoting. This resolves a variant of a conjecture of Gilbert and $\mathrm{Ng}[6]$.

Our result is symbolic in the sense that we assume that addition or subtraction of nonzeros always yields a nonzero result. Gilbert and $\mathrm{Ng}[6]$ also consider what they call exact results; we discuss this further in the conclusion.

A motivation for the current result is its impact on solvers that use the column elimination tree to model factorization in parallel. In solvers like the one described by Gilbert [5] and in the shared memory version of SuperLU [3], the tasks are scheduled dynamically on processors by using the precedence given by the column elimination tree. Our result shows that, in fact, for every strong Hall nonzero structure there is a matrix for which every dependency in the column elimination tree is a real constraint on the order of computation of the columns of the factor.

The next section presents background results and notation used in the paper. Section 3 introduces new results on the structure of the matrix during elimination. These results help to prove the all-at-once structure prediction of the column elimination tree. Section 4 concludes the paper.

\section{Background}

Let $A=\left(a_{r c}\right)$ be a square, possibly unsymmetric, sparse $n \times n$ matrix which is to be factored as $P A=L U$ using partial pivoting.

In the following we introduce the commonly used tree and graph structures, the strong Hall property, a previously published theorem and lemma that will subsequently be used in our proofs. Most of our notation is similar or identical to that of Gilbert and $\mathrm{Ng}[6]$.

The column intersection graph $G_{\cap}(A)$ is undirected and has $n$ vertices (one for each column) and an edge $(i, j)$ if there is an $r$ such that $a_{r i} \neq 0$ and $a_{r j} \neq 0$. The directed graph $G(A)$ has $n$ vertices and an edge $(i, j)$ for each nonzero element $a_{i j}$. The bipartite graph $H(A)$ has $2 n$ vertices (one for each row and one for each column) and an edge $\left(r^{\prime}, c\right)$ whenever $a_{r c}$ is nonzero. In the bipartite graph, we use primes on the names of row vertices. 
For any graph $G$ and vertex $v$, we write $\operatorname{Adj}(v, G)$ to represent the set of vertices $w$ such that $(v, w)$ is an edge of $G$.

The elimination tree structure (etree for short) was first introduced for the Cholesky factorization of symmetric positive definite matrices [8]. If $L$ is the Cholesky factor of the SPD matrix $A$, then this tree has $n$ vertices, and $k$ is the parent of $j$ if and only if $k=\min \left\{r>j: l_{r j} \neq 0\right\}$. Later the elimination tree was adapted to the LU factorization with partial pivoting [5]; the column elimination tree is the elimination tree of the column intersection graph $G_{\cap}(A)$, or equivalently the elimination tree of $A^{T} A$ if there is no numerical cancellation when computing $A^{T} A$.

A strong Hall graph is a bipartite graph with $m$ rows and $n$ columns that has the strong Hall property [2,6]: every set of $k$ column vertices is adjacent to at least $k+1$ row vertices, for all $1 \leq k<n$. A square matrix has the strong Hall property if and only if it is fully indecomposable matrix, that is, there are no two permutations $P$ and $Q$ such that $P A Q$ is block triangular.

Before introducing the necessary theorem and lemma, let us elaborate on an additional definition, that of a sequence of bipartite graphs which model the structure of $L$ and $U$ during the elimination. Let $H_{0}=H(A)$ be the bipartite graph of $A$. Suppose $a_{r c}$ is nonzero and is chosen as pivot at step 1 . The deficiency of the edge $\left(r^{\prime}, c\right)$ of $H_{0}$ is defined as the set of edges :

$$
\left\{\left(i^{\prime}, j\right): c \in \operatorname{Adj}\left(i^{\prime}, H_{0}\right), j \in \operatorname{Adj}\left(r^{\prime}, H_{0}\right) \text {, and } j \notin \operatorname{Adj}\left(i^{\prime}, H_{0}\right)\right\}
$$

It corresponds to the zero elements of $A$ that become nonzero when $a_{r c}$ is used as a pivot in Gaussian elimination.

Knowing the sequence of pivoting elements $\left(r_{1}^{\prime}, c_{1}\right),\left(r_{2}^{\prime}, c_{2}\right), \ldots,\left(r_{n-1}^{\prime}, c_{n-1}\right)$, we can construct a sequence of bipartite graphs $H_{0}, H_{1}, \ldots, H_{n}$, where $H_{i}$ describes the structure of the $(n-i) \times(n-i)$ Schur complement remaining after step $i$. The bipartite graph $H_{i}$ of the $(n-i) \times(n-i)$ submatrix that remains after eliminating $\left(r_{i}^{\prime}, c_{i}\right)$ is obtained as follows: delete from $H_{i-1}$ vertices $r_{i}^{\prime}$ and $c_{i}$ and all edges incident to them, then add the edges in the deficiency of $\left(r_{i}^{\prime}, c_{i}\right)$. The bipartite flled graph $H^{+}(A)$ is the bipartite graph containing all the edges of all $H_{i}$.

If the diagonal elements of $A$ are nonzero, and the pivots are chosen in the order $\left(1^{\prime}, 1\right),\left(2^{\prime}, 2\right), \ldots,\left(n^{\prime}, n\right)$, then we write $G^{+}(A)$ for the filled graph of $A$, which is obtained from $H^{+}(A)$ by merging each row vertex $v^{\prime}$ with its corresponding column vertex $v$. The filled column intersection graph $G_{\cap}^{+}(A)$ is the filled graph of the column intersection graph of $A$, that is, $G^{+}\left(G_{\cap}(A)\right)$.

With these definitions at hand we now mention two results on which ours is based.

Theorem 1 [Gilbert and $\mathrm{Ng}$ [6]/ Let $H_{0}$ be a bipartite graph and let $\left(r^{\prime}, c\right)$ be an edge of $H_{0}$. Let $H_{1}$ be the bipartite graph resulting from the elimination of edge $\left(r^{\prime}, c\right)$. If $H_{0}$ has the strong Hall property, then $H_{1}$ also has the strong Hall property.

For the following lemma (called the fill path lemma), a path is a sequence of edges $P=\left[\left(v_{0}, v_{1}\right),\left(v_{1}, v_{2}\right), \ldots\right.$, $\left.\left(v_{p-1}, v_{p}\right)\right]=\left[v_{0}, v_{1}, \ldots, v_{p}\right]$ in which all the vertices are distinct. The length of this path $P$ is $p$.

Lemma 1 [Rose, Tarjan and Lueker [7]] Let $G$ be a directed or undirected graph whose vertices are the integers 1 through $n$, and let $G^{+}$be its filled graph. Then $(x, y)$ is an edge of $G^{+}$if and only if there is a path in $G$ from $x$ to $y$ whose intermediate vertices are all smaller than $\min (x, y)$.

\section{Structure prediction and the column elimination tree}

In this section we prove the main result of the paper, which is that every strong Hall nonzero pattern admits a pivoting permutation for which every edge of the column elimination tree corresponds to a symbolic column dependency. We first prove a lemma saying that there is a choice of pivot element such that the first elimination step creates the correct dependency for the first column and also does not change the structure of the filled column intersection graph. We then prove the main theorem by induction.

We make two observations about symbolic elimination. First, the fact that $i+1$ is the least-valued vertex in $H_{i}$ implies that there is no fill-in edge having $i+1$ as an endpoint. This gives us

$$
\begin{aligned}
\operatorname{Adj}\left(i+1, G_{\cap}^{+}\left(H_{i}\right)\right) & =\operatorname{Adj}\left(i+1, G_{\cap}\left(H_{i}\right)\right) \\
& =\left\{v: i+1 \in \operatorname{Adj}\left(t^{\prime}, H_{i}\right) \text { and } v \in \operatorname{Adj}\left(t^{\prime}, H_{i}\right)\right\} .
\end{aligned}
$$


Second, the fill path lemma implies that the vertices in the set $\{i+1\} \cup \operatorname{Adj}\left(i+1, G_{\cap}^{+}\left(H_{i}\right)\right)$ form a complete subgraph.

The next lemma shows that when we pivot on an element that is not the only element in its column, enough fill is added to preserve the structure of the filled column intersection graph. For each vertex $i$, we denote its parent in the column elimination tree by parent [i]; by definition this is $\min \left\{j>i: j \in \operatorname{Adj}\left(i, G_{\Pi}^{+}\left(H_{0}\right)\right)\right\}$.

Lemma 2 Let $H_{0}$ be the structure of a square matrix $A$ with at least two nonzero elements in column 1 . Let $P_{1}$ be the permutation matrix that interchanges row $r^{\prime}$ with row 1 such that the edge (1, parent[1]) of the elimination tree of $G_{\cap}(A)$ corresponds to a nonzero in the upper triangular factor $U$. If $H_{1}$ is the bipartite graph resulting from the elimination of edge $\left(r^{\prime}, 1\right)$, then the filled column intersection graph of $H_{1}$ is obtained from the filled column intersection graph of $H_{0}$ just by deleting vertex 1 and its incident edges. That is,

$$
G_{\cap}^{+}\left(H_{1}\right)=G_{\cap}^{+}\left(H_{0}\right)-\{1\}
$$

Proof We will prove that the deficiency set of $\left(r^{\prime}, 1\right)$ introduces all the edges and preserves all the paths that can disappear by the deletion of row $r^{\prime}$ and column 1 , while constructing the graph $H_{1}$. We will also show that this set does not introduce new edges or new fill paths in $G_{\Pi}^{+}\left(H_{1}\right)$ compared to $G_{\Pi}^{+}\left(H_{0}\right)$.

Let us analyze what happens when adding the deficiency of $\left(r^{\prime}, 1\right)$. Let $S=\operatorname{Adj}\left(1, H_{0}\right)$ be the set of row indices of nonzeros in column 1 . From the theorem statement, recall that column 1 is adjacent to at least two row vertices, so that $r^{\prime}$ is not the only element in set $S$.

By using the definition of the deficiency of $\left(r^{\prime}, 1\right)$, for each $t^{\prime} \in S$ such that $t^{\prime} \neq r^{\prime}$ and for each edge $v \neq 1$ adjacent to $r^{\prime}$ in $H_{0}$, we see that $v$ belongs to $\operatorname{Adj}\left(t^{\prime}, H_{1}\right)$. For each two vertices $v_{1}, v_{2} \in \operatorname{Adj}\left(t^{\prime}, H_{1}\right)$, by using the definition of the column intersection graph, we see that $\left(v_{1}, v_{2}\right)$ is an edge of $G_{\cap}\left(H_{1}\right)$.

Let make an analysis depending on the origin of vertices $v_{1}, v_{2}$. First, if $v_{1}, v_{2}$ are adjacent to $r^{\prime}$ in $H_{0}$, (that is $\left.v_{1}, v_{2} \in \operatorname{Adj}\left(r^{\prime}, H_{0}\right)\right)$ the fact that $\left(v_{1}, v_{2}\right)$ is an edge of $G_{\cap}\left(H_{1}\right)$ proves that the deletion of the row $r^{\prime}$ does not change the structure of $G_{\cap}\left(H_{1}\right)$ compared to the structure of $G_{\cap}\left(H_{0}\right)$.

Second, if $v_{1} \in A d j_{H_{0}}\left(r^{\prime}\right)$ and $v_{2} \in A d j_{H_{0}}\left(t^{\prime}\right)$, then $v_{1}$ and $v_{2}$ are both adjacent to 1 in the column intersection graph of $H_{0}$, so $v_{1}, v_{2} \in \operatorname{Adj}\left(1, G_{\cap}\left(H_{0}\right)\right)$. By using the observation at the begining of this section, we see that $\left(v_{1}, v_{2}\right)$ belongs to $G_{\Pi}^{+}\left(H_{0}\right)$. This proves that the deficiency set does not introduce new edges in $G_{\Pi}^{+}\left(H_{1}\right)$.

Using this analysis of edges introduced in $H_{1}$, we can easily check that

$$
\operatorname{Adj}\left(1, G_{\cap}\left(H_{0}\right)\right)-\{v\} \subseteq \operatorname{Adj}\left(v, G_{\cap}\left(H_{1}\right)\right), \forall v \in \operatorname{Adj}\left(r^{\prime}, H_{0}\right), v \neq 1 .
$$

Suppose that $\left[x_{1}, \ldots, x_{r}\right], r>2$ is a fill path in $G_{\Pi}^{+}\left(H_{0}\right)$ and has 1 as an intermediate vertex. This means that $x_{k}<\min \left\{x_{1}, x_{r}\right\}$ for all $k=2, \ldots, r-1$, and the edge $\left(x_{1}, x_{r}\right)$ belongs to $G_{\Pi}^{+}\left(H_{0}\right)$. Suppose that $x_{k}=1, k>1, k<r$. By using relation (4), we see that $x_{k-1}, x_{k+1} \in \operatorname{Adj}\left(\operatorname{parent}[1], G_{\cap}\left(H_{1}\right)\right)$. If $x_{k-1}=$ parent[1] or $x_{k+1}=$ parent[1], it is evident that the fill path is preserved. Otherwise, vertex 1 can be replaced by parent[1] in the path $\left[x_{1}, \ldots, x_{k-1}\right.$, parent[1], $\left.x_{k+1}, \ldots, x_{r}\right]$. By using the definition of the column etree, we see that $x_{k-1}, x_{k+1} \geq$ parent $[1]$, and this shows that $\left[x_{1}, \ldots, x_{r}\right]$ is a fill path in $G_{\Pi}^{+}\left(H_{1}\right)$. This proves that all the fill paths are preserved in $G_{\cap}^{+}\left(H_{1}\right)$ and no new fill path is introduced.

This completes the proof of the lemma.
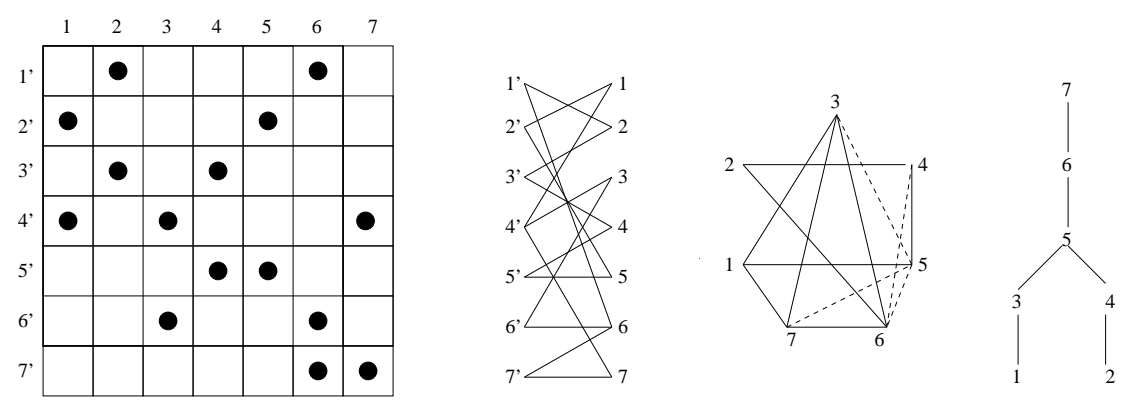

Figure 1: Matrix example $A$, the bipartite graph $H_{0}$, the filled column intersection graph $G_{\Pi}^{+}\left(H_{0}\right)$ and its column elimination tree.

Figure 1 shows a matrix example $A$, its bipartite graph $H_{0}$, followed by the filled column intersection graph $G_{\cap}^{+}\left(H_{0}\right)$ with its column elimination tree. Figure 2 presents the permuted matrix $P_{1} A$, the bipartite graph $H_{1}$ 
with its filled column intersection graph $G_{\cap}^{+}\left(H_{1}\right)$ and the corresponding column elimination tree. Finally, the figure 3 shows the permuted matrix $P A$ and its column intersection graph $G_{\cap}(L+U)$.
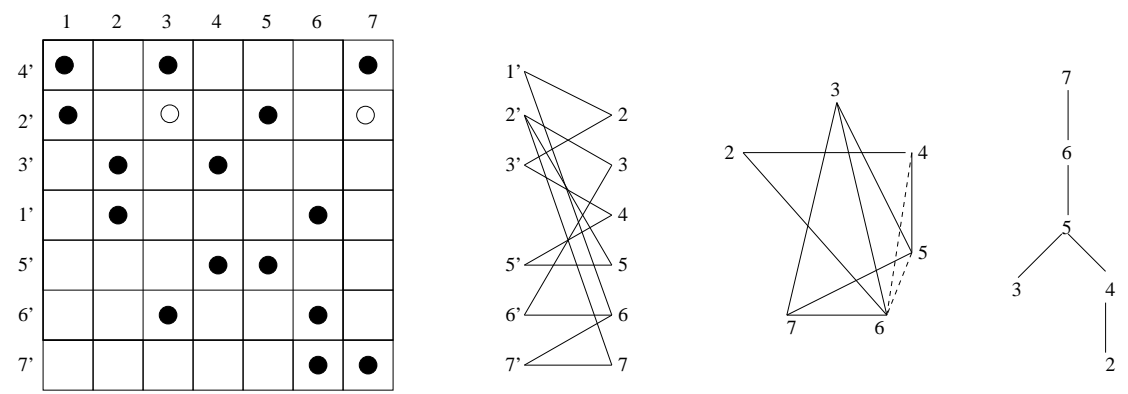

Figure 2: Matrix $P_{1} A$, the bipartite graph $H_{1}$, the filled column intersection graph $G_{\cap}^{+}\left(H_{1}\right)$ and its column elimination tree.

Consider the elimination of edge $\left(4^{\prime}, 1\right)$ in matrix example $A$, figure 1 . The vertices 3 and 7 are adjacent to $4^{\prime}$ in the bipartite graph $H_{0}$. Deleting the row $4^{\prime}$ has as consequence that the edge $(3,7)$ dissapears from $G_{\Pi}^{+}\left(H_{1}\right)$. By adding the deficiency of $\left(4^{\prime}, 1\right)$, the edge $(3,7)$ is introduced in $G_{\Pi}^{+}\left(H_{1}\right)$ due to row vertex $2^{\prime}$. Now consider the vertex 3 adjacent to vertex $4^{\prime}$ and the vertex 5 adjacent to vertex $2^{\prime}$ in the bipartite graph $H_{0}$. By the permutation of row $4^{\prime}$ with row $1^{\prime}$, the edge $(3,5)$ is introduced in the column intersection graph $G_{\cap}^{+}\left(H_{1}\right)$. However, we remark that $(3,5)$ was already present in the column intersection graph $G_{\cap}^{+}\left(H_{0}\right)$. Finally, we consider the fill path $\left[\begin{array}{llll}5 & 1 & 3 & 6\end{array}\right]$ in $G_{\cap}^{+}\left(H_{0}\right)$ which is preserved in $G_{\cap}^{+}\left(H_{1}\right)$ in a compact form $\left[\begin{array}{lll}5 & 3 & 6\end{array}\right]$.

The next theorem is the main result of this paper. It proves the conjecture of Gilbert and $\mathrm{Ng}$ [6] in the symbolic sense, that, if we assume that zeros are introduced only by explicit elimination and not by cancellation.

Theorem 2 Let $A$ be an unsymmetric square sparse matrix having the strong Hall property. There is a permutation $P$ such that every edge of the elimination tree of $G_{\cap}(A)$ corresponds to a nonzero in the upper triangular factor $U$ in symbolic sense, when the factorization $P A=L U$ is computed.

ProOF We will prove this by induction. Let $H_{0}=H(A)$ be the bipartite graph of $A$.

Initial phase: We show that there exists a permutation $P_{1}$ such that the element $u_{1, \text { parent[1] }}$ is nonzero.

Using relation (1), we see that $\left(1\right.$, parent[1]) belongs to $G_{\cap}\left(H_{0}\right)$. There exists a row vertex $r_{1}^{\prime}$ such that $\left(r_{1}^{\prime}, 1\right)$ and $\left(r_{1}^{\prime}\right.$, parent[1]) are edges of $H_{0}$. We choose $r_{1}^{\prime}$ as pivot, and $P_{1}$ describes this permutation. Row 1 is interchanged with row $r_{1}^{\prime}$; therefore the element $u_{1 \text {,parent[1] }}$ is nonzero.

Induction phase $(m-1 \rightarrow m)$ : We suppose that there is a sequence of permutations $P_{m-1}, \ldots, P_{1}$ such that for all $k=1 \ldots m-1, u_{k, \text { parent }[k]}$ is nonzero. We show that there is a permutation $P_{m}$ such that the element $u_{m, \text { parent }[m]}$ is nonzero.

According to Theorem 1, at each elimination step $k$, the bipartite graph $H_{k}$ is strong Hall because $H_{k-1}$ is. In particular, this means that at each elimination step we have at least two elements as choices to pivot on (column vertex $k$ is adjacent to at least two row vertices in the graph $H_{k-1}$ ).

Therefore, Lemma 2 applies, and says that at each elimination step $k$, the structure of the filled column intersection graph is preserved:

$$
G_{\cap}^{+}\left(H_{k}\right)=G_{\cap}^{+}\left(H_{k-1}\right)-\{k\} \quad 1 \leq k<m .
$$

This relation shows that the induction hypothesis has as direct consequence that the structure of the filled column intersection graph was preserved until this step $m$ of elimination. We can deduce that $(m$, parent $[m])$ belongs to $G_{\cap}^{+}\left(H_{m-1}\right)$. Even more, relation (1) says that this edge belongs to $G_{\cap}\left(H_{m-1}\right)$.

Thus, there is some vertex $r_{m}^{\prime}$ such that $\left(r_{m}^{\prime}, m\right)$ and $\left(r_{m}^{\prime}\right.$, parent $\left.[m]\right)$ are edges of $H_{m-1}$. We choose $r_{m}^{\prime}$ as pivot, and let $P_{m}$ describe this permutation. The permutation of the row $m$ with the row $r_{m}^{\prime}$ will make the element $u_{m \text {,parent }[m]}$ be nonzero.

Let $P=P_{n-1}, \ldots, P_{1}$ be the permutation matrix that includes the $n-1$ row interchanges. We have proved that every edge of the column elimination tree corresponds to a symbolic nonzero in the upper triangular factor $U$, when the factorization $P A=L U$ is computed with partial pivoting.

This completes the proof of the theorem. 

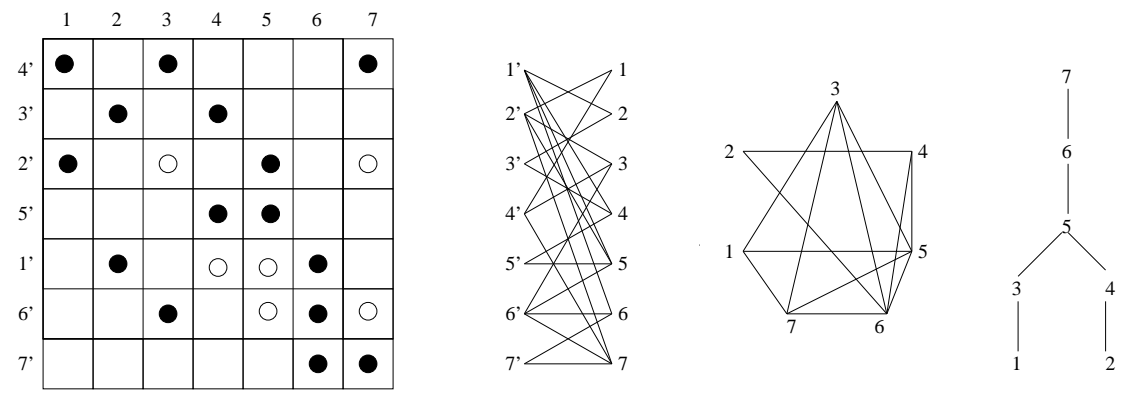

Figure 3: The permuted factorized matrix $P A$, the bipartite filled graph $H^{+}(A)$, the column intersection graph $G_{\cap}(L+U)$ and its column elimination tree.

\section{Concluding remarks}

The main result of this paper is Theorem 2, which gives an all-at-once structure prediction result, under the assumption that the matrix $A$ is strong Hall. In the proof, we showed that if at each elimination step $k$ the element $u_{k, \text { parent }[k]}$ is nonzero, the structure of the filled column intersection graph is preserved during the elimination. One way to interpret this result is that we learn the structure of $U$ one row at a time during elimination, but we never learn anything more about the uncomputed rows of $U$ than we knew from $G_{\cap}^{+}$at the beginning. In other words, the elimination does not give progressively more information about $U$.

We remark that, in the proof of Theorem 2, the strong Hall property was used in only one place for each elimination step. We used the strong Hall property to conclude that at each step (except the last), there is always a choice of at least two elements to pivot on. One could ask whether the strong Hall property is necessary as well as sufficient for this.

Our result is symbolic, in the sense that we assume that during Gaussian elimination the result of adding or subtracting two nonzeros is never zero. A stronger result would be what Gilbert and $\mathrm{Ng}$ [6] called exact, which would assume only that the nonzero values in $A$ were algebraically independent from each other; in other words, it would assume that any computed zeros were due to combinatorial properties of the nonzero structure rather than to coincidence in choice of values. We do not know whether the exact version of our main theorem holds or not, though we conjecture that it does. An exact version holds, for example, for the class of strong Hall matrices with exactly two nonzeros in every row and every column, because every elimination step creates exactly one new nonzero and that nonzero is algebraically independent of the other remaining nonzeros.

We conclude by mentioning an open problem: what is the case for non strong Hall matrices, either for the elimination tree or for the structures of $L$ and $U$ ? In this case, it is known that $G_{\Pi}^{+}(A)$ may not be a tight bound for $U$. Is there a tight bound on $U$ ? If so, does it share the property that there is no new information revealed during the elimination except the structure of the current row of $U$ ?

\section{References}

[1] R. A. Brualdi and H. J. Ryser. Combinatorial Matrix Theory. Cambridge University Press, 1991.

[2] R. A. Brualdi and B. A. Shader. Strong Hall matrices. SIAM J. Matrix Anal. Appl., 15:359-365, 1994.

[3] J. W. Demmel, J. R. Gilbert, and X. S. Li. A Parallel Supernodal Approach to Sparse Partial Pivoting. SIAM J. Matrix Anal. Appl., 20(3):720-755, 1999.

[4] A. George and E. Ng. Symbolic factorization for sparse gaussian elimination with partial pivoting. SIAM J. Sci. Stat. Comput., 8(6):877-898, 1987.

[5] J. R. Gilbert. An efficient parallel sparse partial pivoting algorithm. Technical Report 88/45052-1, Christian Michelsen Institute, 1988.

[6] J. R. Gilbert and E. G. Ng. Predicting Structure in Nonsymmetric Sparse Matrix Factorizations. In A. George, J. R. Gilbert, and J. W. H. Liu, editors, Graph Theory and Sparse Matrix Computation, pages 107-139. Springer Verlag, 1994.

[7] D. J. Rose and R. E. Tarjan. Algorithmic aspects of vertex elimination on directed graphs. SIAM J. Appl. Math., 34(1):176-197, 1978.

[8] R. Schreiber. A new implementation of sparse Gaussian elimination. ACM Trans. Math. Software, 8:256-276, 1982. 
Unité de recherche INRIA Lorraine

LORIA, Technopôle de Nancy-Brabois - Campus scientifique

615, rue du Jardin Botanique - BP 101 - 54602 Villers-lès-Nancy Cedex (France)

Unité de recherche INRIA Rennes : IRISA, Campus universitaire de Beaulieu - 35042 Rennes Cedex (France)

Unité de recherche INRIA Rhône-Alpes : 655, avenue de l'Europe - 38330 Montbonnot-St-Martin (France)

Unité de recherche INRIA Rocquencourt : Domaine de Voluceau - Rocquencourt - BP 105 - 78153 Le Chesnay Cedex (France)

Unité de recherche INRIA Sophia Antipolis : 2004, route des Lucioles - BP 93 - 06902 Sophia Antipolis Cedex (France) 